\title{
On Using an Automatic Scheme for Obtaining the Convex Hull Defining Inequalities of a Weismantel 0-1 Knapsack Constraint
}

\author{
Laureano F. Escudero \\ Dpto. de Estadística e I.O., U.C.M., and \\ Iberdrola Ingeniería y Consultoría, Spain \\ Araceli Garin \\ DEAIII, Universidad del País Vasco, Bilbao, Spain \\ Gloria Pérez \\ DMAEIO, Universidad del País Vasco, Bilbao, Spain
}

\begin{abstract}
In this short note we obtain the full set of inequalities that define the convex hull of a 0-1 knapsack constraint presented in Weismantel (1997). For that purpose we use our $\mathrm{O}(\mathrm{n})$ procedures for identifying maximal cliques and non-dominated extensions of consecutive minimal covers and alternates, as well as our schemes for coefficient increase based tightening cover induced inequalities and coefficient reduction based tightening general 0-1 knapsack constraints.
\end{abstract}

Key Words: 0-1 program, cover, clique, knapsack constraint, coefficient increase, coefficient reduction.

AMS subject classification: $90 \mathrm{C09}$, $90 \mathrm{C} 10$.

\section{Introdution}

Consider the 0-1 program

$$
\max \left\{d x: A x \leq b, x \in\{0,1\}^{m}\right\},
$$

where $x$ is the m-column vector of the $0-1$ variables, $d$ is the related row vector of the objective function coefficients, $A$ is the coefficient matrix of the constraints and $b$ is the column vector of the right-hand-side (hereafter, $r h s)$. All vectors and the matrix are assumed to have the appropriate dimensions.

Let $I$ and $J$ be the set of indices of the constraints and variables, respectively, and $J_{i}$ be the set of indices of the variables with nonzero coefficient

Received: December 1997; Accepted: March 1998 
in the $i$-th constraint for $i \in I$. (The LP relaxation is the same system (1.1), where each $x_{j}$ is allowed to take any value in the range [0, 1]).

We consider knapsack constraints of the form

$$
a_{r} x \leq b_{r}
$$

where $a_{r}$ is the $r$-th row vector of matrix $A$ and $b_{r}$ is the $r$-th element of rhs $b$. Let $a_{r j}$ be the $j$-th element of vector $a_{r}$; without loss of generality, we assume that $a_{r j}$ and $b_{r}$ are integer and strictly positive, $a_{r j} \leq b_{r} \forall j \in J_{r}$ and $\sum_{j \in J_{r}} a_{r j}>b_{r}$ for any $r \in I$.

Let $n=\left|J_{i}\right|$. The knapsack polytope $P$ is the convex hull of the $0-1$ points satisfying (1.2).

For any subset $V \subseteq J_{i}$, let $P_{V}=\operatorname{con}\left\{x \in\{0,1\}^{V} / \sum_{j \in V} a_{i j} x_{j} \leq b_{i}\right\}$.

For a given constraint system some other system may have exactly the same set of $0-1$ feasible solutions. Such constraints are said to be 0-1 equivalent systems or simply equivalent. See Nemhauser and Wolsey (1988) and Wolsey (1989) for good surveys on equivalent systems. Note that equivalent systems can have quite different intersections with the hypercube $0 \leq x \leq 1$. We say that the system $A^{\prime} x \leq b^{\prime}$ is as tight as the system $A x \leq b$ whenever

$$
\left\{x \in[0,1]: A^{\prime} x \leq b^{\prime}\right\} \subseteq\{x \in[0,1]: A x \leq b\}
$$

We say that $A^{\prime} x \leq b^{\prime}$ is tighter than $A x \leq b$ if the containment (1.3) is proper. We are interested in finding tight formulations for (1.1).

Tight equivalent inequalities can be obtained by using coefficient reduction and coefficient increase techniques. Our methods, see Dietrich et al. (1993), Escudero et al. (1997), and Escudero et al. (1995) (appropriately embedded in branch-and-cut procedures, see Hoffman and Padberg (1991)) may produce reductions of the LP feasible set that are not generally detected by other (so-called myopic) procedures, where only information from the same constraint to replace is exploited, aside the integrality of the variables.

Let two of the most frequent $0-1$ structures that are encountered in real-life models:

Cover. Set of indices of 0-1 variables, say $C$, such that at most $k_{C}$ of such variables can take the value 1 . So, the induced inequality can be 
writen

$$
X(C) \leq k_{C}
$$

where $X(C)=\sum_{j \in C} x_{j}$ and $1 \leq k \leq|C|-1$. We say that cover $C$ is implied by knapsack constraint (1.2) provided that $\sum_{j \in C} a_{i j}>b_{i}$.

A cover $C$ is called a minimal cover implied by knapsack constraint (1.2) if $\sum_{j \in C-\{l\}} a_{i j} \leq b_{i} \forall l \in C$. It is well known (see Balas and Zemel (1978), Padberg (1973) and Weismantel (1997), among others) that the inequality

$$
X(C) \leq|C|-1
$$

is a facet defining inequality for $P_{C}$ if and only if $C$ is a minimal cover.

Clique. A cover with $k=1$, so the induced inequality can be written

$$
X(C) \leq 1 .
$$

Let also the additional definitions.

Extension of a minimal cover. Set of indices of 0-1 variables that includes the indices of the minimal cover plus the indices of the coefficients in the implying knapsack constraint that are not smaller than any coefficient whose index is included in the minimal cover. So, the extension $E(C)$ of the minimal cover $C$ for the $i$-th knapsack constraint can be expressed

$$
E(C)=C \cup\left\{l \in J_{i}: a_{i l} \geq a_{i j}, j \in C\right\} .
$$

See that $X(E(C)) \leq k_{C}$.

Alternate set of a minimal cover. Set of indices of 0-1 variables that are alternatives to the index from the minimal cover with the smallest coefficient in the implying knapsack constraint, such that the new set is also a minimal cover. So, the alternate set $A(C)$ of the minimal cover $C$ for the $i$-th knapsack constraint can be expressed

$$
A(C)=\left\{l \in J_{i}: a_{i l} \leq a_{i j}, j \in C: \sum_{j \in C \cup\{l\}-p} a_{i j}>b_{i}\right\}
$$

where $p \in C$ such that $a_{p} \leq a_{j} \forall j \in C$. Note that $X(E(A(C))) \leq k_{C}$.

The scope of this brief note is to show how the full set of inequalities presented by Weismantel (1997) to define the convex hull of the 0-1 
points satisfying a 0-1 knapsack constraint (1.2), can be derived by using successively our set of procedures for: (1) identifying maximal cliques and non-dominated extensions of consecutive minimal covers and alternates, see Escudero et al. (1995); (2) coefficient increase based tightening cover induced inequalities, see Dietrich et al. (1994) and Escudero and Muñoz (1998); and (c) coefficient reduction based tightening general 0-1 knapsack constraints.

The note is organized as follows. Section 2 introduces our basic principles for coefficient increase and reduction based methods. Section 3 gives the Weismantel 0-1 knapsack constraint and the related set of convex hull defining inequalities, and section 4 describes our scheme to derive the set of the inequalities referenced above.

\section{Basic Tightening Schemes}

\subsection{Coefficient Increase}

Consider the knapsack constraint (1.2) and let $C \subseteq J$ be a cover whose induced inequality (1.4) is satisfied by any feasible solution for (1.1). If there exists $l \in C$ such that $S_{r}^{l}<b_{r}$ for $r \in I$, where

$$
S_{r}^{l}=\max \left\{\sum_{j \in J_{r}} a_{r j} x_{j} / \sum_{j \in J_{i}} a_{i j} x_{j} \leq b_{i} \forall i \in I, x_{l}=1, x_{j} \in\{0,1\} \forall j \in J\right\},
$$

we define

$$
\begin{aligned}
\Delta_{r, C}^{+j} & =\left(b_{r}-S_{r}^{j}\right) / k_{C}, \forall j \in C \\
\bar{a}_{r j} & = \begin{cases}a_{r j}+\Delta_{r, C}^{+j} & \forall j \in C \\
a_{r j} & \forall j \in J_{r} \backslash C\end{cases}
\end{aligned}
$$

As a result, let the constraint

$$
\sum_{j \in J_{r} \cup C} \bar{a}_{r j} x_{j} \leq b_{r}
$$

Theorem 2.1. Replacing the r-th knapsack constraint (1.2) by (2.3) in (1.1), results in a 0-1 equivalent and as-tight-as formulation. The new formulation is tighter than the original one if and only if (2.3) is a nonredundant constraint. 
Proof: See Escudero and Muñoz (1997).

\subsection{Coefficient Reduction}

Consider the knapsack constraint (1.2) and let $C \subseteq J$ be a cover whose induced inequality (1.4) is satisfied by any feasible solution for (1.1). If $R_{r, C}<b_{r}$ for $r \in I$, where

$$
\begin{aligned}
R_{r, C}=\max \left\{\sum_{j \in J_{r}} a_{r j} x_{j} / \sum_{j \in J_{i}} a_{i j} x_{j} \leq b_{i} \forall i \in I,\right. \\
\left.\sum_{j \in C} x_{j} \leq k_{C}-1, x_{j} \in\{0,1\} \forall j \in J\right\},
\end{aligned}
$$

we define

$$
\begin{aligned}
\Delta_{r, C}^{-}= & b_{r}-R_{r, C} \\
\bar{a}_{r j}= & \begin{cases}a_{r j}-\Delta_{r, C}^{-} / k_{C} & \forall j \in C \\
a_{r j} & \forall j \in J_{r} \backslash C\end{cases} \\
& \bar{b}_{r}=b_{r}-\Delta_{r, C}^{-}
\end{aligned}
$$

As a result, let the constraint

$$
\sum_{j \in J_{r} \cup C} \bar{a}_{r j} x_{j} \leq \bar{b}_{r}
$$

Theorem 2.2. Replacing the $r$-th knapsack constraint (1.2) by (2.6) and (1.4) in (1.1), results in a 0-1 equivalent and as-tight-as formulation. The new formulation is tighter than the original one if and only if there exists a feasible solution for the LP relaxation of (1.1) that verifies any of the two following conditions:

$$
\begin{aligned}
& b_{r}-\sum_{j \in J_{r}} a_{r j} x_{j}<\frac{\Delta_{r, C}^{-}}{k_{C}}\left(k_{C}-\sum_{j \in C} x_{j}\right) \\
& \sum_{j \in C} x_{j}>k_{C}
\end{aligned}
$$

Proof: See Escudero and Muñoz (1998). 


\section{Weismantel 0-1 Knapsack Constraint}

The 0-1 knapsack constraint subject of our analysis is as follows, see Weismantel (1997).

$$
x_{1}+x_{2}+x_{3}+x_{4}+3 x_{5}+4 x_{6} \leq 4
$$

Table 1 shows the set of the inequalities that define the convex hull of the 0-1 points satisfying (3.1).

\begin{tabular}{|c|c|c|c|c|c|c|c|}
\hline$x_{1}$ & $x_{2}$ & $x_{3}$ & $x_{4}$ & $x_{5}$ & $x_{6}$ & & \\
\hline & & & & 1 & 1 & $\leq$ & 1 \\
& & & 1 & & 1 & $\leq$ & 1 \\
& & 1 & & & 1 & $\leq$ & 1 \\
& 1 & & & & 1 & $\leq$ & 1 \\
1 & & & & & 1 & $\leq$ & 1 \\
\hline & & 1 & 1 & 1 & 2 & $\leq$ & 2 \\
& 1 & & 1 & 1 & 2 & $\leq$ & 2 \\
1 & & & 1 & 1 & 2 & $\leq$ & 2 \\
& 1 & 1 & & 1 & 2 & $\leq$ & 2 \\
1 & & 1 & & 1 & 2 & $\leq$ & 2 \\
1 & 1 & & & 1 & 2 & $\leq$ & 2 \\
\hline & 1 & 1 & 1 & 2 & 3 & $\leq$ & 3 \\
1 & & 1 & 1 & 2 & 3 & $\leq$ & 3 \\
1 & 1 & & 1 & 2 & 3 & $\leq$ & 3 \\
1 & 1 & 1 & & 2 & 3 & $\leq$ & 3 \\
\hline
\end{tabular}

Table 1: Convex hull defining inequalities

\section{Deriving the set of Convex Hull Defining Inequalities}

By using our scheme for identifying maximal cliques, see Dietrich et al. (1994), the following cliques are obtained: $C_{j}=\{j, 6\}$ for $j=1,2, \ldots, 5$. 
So, the following clique inequalities are identified

$$
\begin{aligned}
& x_{5}+x_{6} \leq 1 \\
& x_{4} \quad+x_{6} \leq 1 \\
& x_{3} \quad+x_{6} \leq 1 \\
& x_{2} \quad+x_{6} \leq 1 \\
& x_{1} \quad+x_{6} \leq 1 \text {. }
\end{aligned}
$$

Our scheme for identifying minimal covers and its extensions and alternates, see Dietrich et al. (1994), gives the following covers:

1. Minimal cover: $\{3,4,5\}$ with $k_{C}=2$

Extension: $\{3,4,5,6\}$

Alternate set to index 3: $\{1,2\}$

So, the following covers inequalities are identified for $k_{C}=2$ :

$$
\begin{aligned}
& x_{3}+x_{4}+x_{5}+x_{6} \leq 2 \\
& x_{2} \quad+x_{4}+x_{5}+x_{6} \leq 2 \\
& x_{1}+x_{4}+x_{5}+x_{6} \leq 2
\end{aligned}
$$

2. Minimal cover: $\{2,3,5\}$ for $k_{C}=2$

Extension: $\{2,3,5,6\}$

Alternate set to index 2: $\{1\}$

So, the following cover inequalities are identified for $k_{C}=2$ :

$$
\begin{array}{llllll} 
& x_{2} & +x_{3} & +x_{5} & +x_{6} & \leq 2 \\
x_{1} & & +x_{3} & +x_{5} & +x_{6} & \leq 2
\end{array}
$$

3. Minimal cover: $\{1,2,5\}$ for $k_{C}=2$

Extension: $\{1,2,5,6\}$

So, the following cover inequality is identified for $k_{C}=2$ :

$$
x_{1}+x_{2}+x_{5}+x_{6} \leq 2
$$

By using our scheme described in Escudero et al. (1997), see also Dietrich and Escudero (1992), the six above cover inequalities are reinforced. For 
this purpose the clique inequalities (4.1) are used to tighten the coefficient of $x_{6}$, such that the new constraints are as follows:

$$
\begin{array}{lllllll} 
& & x_{3} & +x_{4} & +x_{5} & +2 x_{6} & \leq 2 \\
& x_{2} & & x_{4} & +x_{5} & +2 x_{6} & \leq 2 \\
x_{1} & & & x_{4} & +x_{5} & +2 x_{6} & \leq 2 \\
& & & & & \\
& x_{2} & +x_{3} & & +x_{5} & +2 x_{6} & \leq 2 \\
x_{1} & & +x_{3} & & +x_{5} & +2 x_{6} & \leq 2 \\
x_{1} & +x_{2} & & & +x_{5} & +2 x_{6} & \leq 2 .
\end{array}
$$

Let us use our coefficient reduction scheme given in Escudero et al (1995), see also Dietrich et al. (1993), for deriving the other facet defining inequalities for the original 0-1 knapsack constraint (3.1). To begin with let us drop the variable $x_{1}$ from (3.1). The new constraint will be tightened by using the clique $\{5,6\}$. So, the auxiliary problem to solve can be expressed

$$
\begin{array}{cccccccc}
R=\text { max } & & x_{2} & +x_{3} & +x_{4} & +3 x_{5} & +4 x_{6} & \\
\text { s.t. } & x_{1} & +x_{2} & +x_{3} & +x_{4} & +3 x_{5} & +4 x_{6} & \leq 4 \\
& & & & & x_{5} & +x_{6} & \leq 0 \\
& & & & & x_{j} & \in\{0,1\} & \forall j .
\end{array}
$$

So, $R=3$ and, then, the tighter constraint can be expressed

$$
x_{2}+x_{3}+x_{4}+2 x_{5}+3 x_{6} \leq 3 .
$$

By using successively the same approach for $x_{2}, x_{3}$ and $x_{4}$ as it has been done for $x_{1}$ above, the new constraints are as follows:

$$
\begin{array}{lllllll}
x_{1} & & +x_{3} & +x_{4} & +2 x_{5} & +3 x_{6} & \leq 3 \\
x_{1} & +x_{2} & & +x_{4} & +2 x_{5} & +3 x_{6} & \leq 3 \\
x_{1} & +x_{2} & +x_{3} & & +2 x_{5} & +3 x_{6} & \leq 3
\end{array}
$$

See that the inequalities (4.1) - (4.4) plus the bounding constraints $0 \leq x_{j} \leq 1$, are exactly the inequalities shown in table 1 .

\section{Conclusion}

The set of inequalities that define the convex hull of the points satisfying a given 0-1 knapsack constraint has been derived by using an automatic scheme for efficient tightening of 0-1 programs. The approach turned out to be very efficient. In fact, it has been successfully used for solving an extensive set of real-life problems, see the given references. 


\section{References}

Balas E. and E. Zemel (1978). Facets of the knapsack polytope from minimal covers. SIAM J. of Applied Mathematics 39, 119-148.

Dietrich B.L. and L.F. Escudero. (1992) On tightening cover induced inequalities. European Journal of Operational Research 60, 335-343.

Dietrich B.L., L.F. Escudero and F. Chance. (1993) Efficient reformulation for 0-1 programs, methods and computational results. Discrete Applied Mathematics 42, 147-175.

Dietrich B.L., L.F. Escudero, A. Garín and G. Pérez.(1994) O(n) procedures for identifying maximal cliques and non-dominated extensions of consecutive minimal covers and alternates. TOP Trabajos de Investigación Operativa 1, 139160.

Escudero L.F., A. Garín and G. Pérez.(1999) O(nlogn) procedures for tightening cover inequalities. European Journal of Operational Research 13, 676-687.

Escudero L.F., S. Martello and P. Toth. (1995) A framework for tightening 0-1 programs based on extensions of pure 0-1 KP and SS problems, in: E. Balas and $\mathrm{J}$. Clausen (eds.). Integer programming and combinatorial optimization (Springer-Verlag, Berlin,) 110-123.

Escudero L.F. and S. Muñoz. (1998) On characterizing tighter formulations for 0-1 programs, European Journal of Operational Research 106, 172-176.

Hoffman K.L. and M.W. Padberg. (1991) Improving LP representations of zeroone linear programs for branch-and-cut. SIAM Journal on Computing 3, 121128.

Nemhauser G.L. and L.A. Wolsey. (1988) Integer and combinatorial optimization. John Wiley, N.Y.

Padberg M.W. (1973) On the facial structure of the set packing polyhedra. Mathematical Programming 5, 199-216.

Weismantel R. (1997), On the 0-1 knapsack polytope. Mathematical Programming 77, 49-68.

Wolsey L.A. (1989) Tight formulations for mixed integer programming. A survey. Mathematical Programming 45, 137-191. 